\title{
Increased suppression during punishment applied to the responding member'
}

\author{
Arnold Kaufman \\ UNIVERSITY OF WISCONSIN, MILWAUKEE
}

\begin{abstract}
Abstraet
Subjects responding with the right hand were punished with electric shock on the right and left hands during alternate 4-min. periods. At low and high intensities there was no differential effect, but at moderate intensities punishment to the responding hand produced more suppression than punishment to the non-responding hand.

\section{Problem}

In a recent report on the effects of punishing electric shock, Appel (1963), has pointed to a number of discrepancies between results obtained by Azrin (1960), and Appel (1961), as well as Storms etal. (1962). Azrin has consistently found that when low or intermediate shock intensities are employed, there is a uniform initial suppression of responding followed by later recovery which is inversely related to the shock intensity being applied. With extremely high intensities, Azrin reports virtually complete suppression and no later recovery. Appel, on the other hand, reports that initial suppression is greater when higher shock intensities are employed, but finds no recovery at any intensity. As Appel (1961) points out, one of the major differences between these studies lies in the method of shock delivery. In Azrin's implant method, the pigeon responds with its beak and is shocked near the tail. This method has led to maximum recovery of rate. With Appel's methods, as well as those of others reporting no recovery from shock punishment, the animal responds with a paw and closes the shock circuit from the grid floor to the lever when the paw makes contact with the lever. With this method, not only does the current flow through the major portion of the animal's body, but it is also possible that maximum stimulation occurs at the paw as the animal touches the bar. One possibility, therefore, is that a major difference may exist between the implant and grid-lever methods in that with grid-lever shock the animal is being shocked on the part of the body involved in the response; i.e., directly on the responding paw. With the implant method, the animal responds with its beak, but is shocked on the part of the body furthest removed from the responding member.

The present investigation was designed to explore the possibility that point of application of shock punishment, especially relative to the responding member, is a variable which leads to differential suppressive effects. More specifically, this study is concerned with the differences in initial suppressive effects when punishment shock is delivered to the responding as opposed to the non-responding member.

\section{Method}

In the situation studied, four human Ss, all females, were paid on the basis of the total number of responses made per session. Throughout all sessions, whether punished or not, responding on a push button manipulandum was paid at the rate of one cent for 50 responses (VR 50). Each 52-min. session consisted of alternating 4-min. periods of unpunished and punished responding. In odd numbered periods $\mathrm{S}$ could respond or not but was never shocked. In even numbered periods each response was followed immediately by a $40 \mathrm{msec}$., $60 \mathrm{cps}$ pulse. $\mathrm{S}$ was limited to always responding with the fingers of the right hand, and shock electrodes were placed on the index fingers of both the right and left hands. An effort was made to place the electrodes on the same part of the index finger on both hands, so as to minimize any qualitative differences in the shock stimulation which might otherwise be due to stimulating cutaneous areas with markedly different distributions of pain receptors. Within the sequence of even numbered (shock) periods, Ss alternately received shock on either hand. Three Ss received shock on the left hand in the 2nd, 6th, and 10th periods, with shock to the responding hand in the $4 \mathrm{th}, 8 \mathrm{th}$, and $12 \mathrm{th}$ periods, while the fourth $\mathrm{S}$ was exposed to shock on the right (responding) hand first and then alternated from hand to hand in the remaining shock periods. All Ss were exposed to 10 daily sessions over a two week period, with punishment shock intensity varying among five values from day to day. The punishment intensities used, expressed in ma and in the order of their daily presentation, were: (1) $0 \mathrm{ma}$; (2) $1.6 \mathrm{ma}$; (3) $4.8 \mathrm{ma}$; (4) $3.2 \mathrm{ma}$; (5) $6.4 \mathrm{ma}$; (6) $4.8 \mathrm{ma}$; (7) $1.6 \mathrm{ma}$; (8) $6.4 \mathrm{ma}$; (9) $0 \mathrm{ma}$; (10) $3.2 \mathrm{ma}$. Thus, each value was presented twice, once during the first five sessions and once during the second five sessions.

\section{Hesults}

At all levels of shock intensity, as indicated by Fig. 1, responding during the no-shock periods remained quite stable and at a high rate, indicating that none of the intensities was severe enough to suppress all responding. Figure 1 contains data taken only from the last five sessions, by which time the no-shock rate had stabilized. In the first five sessions, the no-shock rate showed a steady increase from day to day and there was some variability in response to the shock although the overall effect of increased suppression during punishment of the responding hand was apparent. By the fifth day, the discrimination of the basic pattern of alternating 4-min. periods had stabilized, as had the no-shock rate, and the data in Fig. 1 reflects this in the similarity from $\mathrm{S}$ to $\mathrm{S}$. In all cases, there is virtually no punishment effect at $1.6 \mathrm{ma}$, with the left and right hand shock responding falling within the range of variability of the no-shock responding. At $6.4 \mathrm{ma}$, all four Ss are showing almost complete suppression during both left and right hand shock punishment, with no large difference between hands due to this almost complete elimination of responding. Where slight differences do occur as with $\mathrm{S}$ three, they indicate more suppression with shock applied to the responding hand. The large differences in sup- 


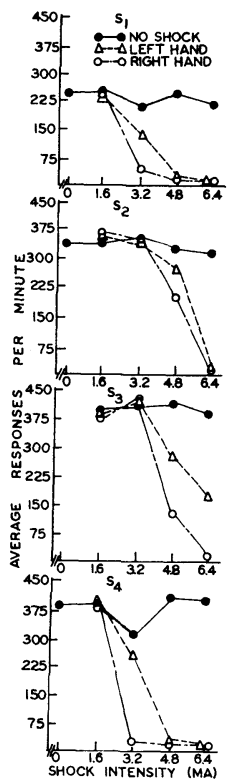

Fig. 1. Average rate of responding for four human subjects during each of five days at a different shock intensity. Responding was limited to the right hand. Button presses were intermittently reinforced with money and shock was delivered after each response during alternate 4-min. periods. In half of the shock periods, punishment was delivered to the right (responding) hand, in the other half to the left (non-responding) hand.

pression come at 3.2 and $4.8 \mathrm{ma}$, with Ss one and four showing a much greater degree of suppression during punishment of the responding hand at $3.2 \mathrm{ma}$, and a minimal difference in the same direction at $4.8 \mathrm{ma}$, at which level responding again has been virtually eliminated. Ss two and three show virtually no shock effect at $3.2 \mathrm{ma}$, but again a large increase in suppression during punishment of the responding hand at $4.8 \mathrm{ma}$.

\section{Diseussion}

The overall effect of point of application, then, is to maximize suppression when the responding member is punished with intensities which do not completely eliminate responding. This finding fits in well with the contradictory results obtained when the implanted electrode technique has been used as opposed to the grid-lever method. In general, the implanted electrode technique, where shock is applied distal to the responding member, has resulted in less suppression and much faster recovery. Similarly, in the present study there was less suppression observed when the non- responding hand was punished. On the other hand, with the grid-lever method, with shock applied directly on or proximal to the responding member, the suppressive effects have been greater at relatively low shock intensities, and recovery does not occur. In the present study suppression was also greater when the responding hand was punished.

It is possible that these results, as with the animal work in this area, may be attributed to hand withdrawal due to muscular tetanization caused by passing a current through the muscles of the responding member. Three observations made in our laboratory seem to rule out this interpretation. First, we have run at least four Ss who have shown no differences between no shock and shock responding, even when intensities as high as $12 \mathrm{ma}$ have been applied to the responding member. Second, our Ss, on termination of the experiment, uniformly report not a physical inability to respond, but rather a greater degree of vacillation and hesitancy just prior to responding. Third, in applying the shock to himself and his assistants, $\mathrm{E}$ has experienced no difficulty in responding under shock at the highest intensities employed in this study. No effects of muscular rigidity were apparent until about $14 \mathrm{ma}$ were utilized, and even then using another finger of the right hand, which was quite common with Ss, removed all difficulty in responding.

The results of this study, then, confirm the findings of previous studies on punishment shock intensity as they apply to initial suppressive effect. With only two sessions at the intensity levels where differential suppression was observed, nothing can be said about recovery. Presumably, with many more sessions at intermediate shock levels, we might expect a faster rate of recovery when punishment is delivered to the non-responding member.

\section{References}

APPEL, J. B. Punishment in the squirrel monkey Sa i m i r i s c i u rea. Science, $1961,133,36$.

APPEL, J. B. Punishment and shock intensity. Science, 1963, 141, 528-529.

AZRIN, N. Sequential effects of punishment. Science, 1960, 131, 605-606.

STORMS, L. H., BOROCZI, G., \& BROEN, W. E. JR. Punishment inhibits an instrumental response in hooded rats. Science, 1962, $135,1133-1134$

\section{Note}

1. Supported by grant RF 62:616 from the Graduate School of the University of Wisconsin. I thank Dr. Harry L. Madison, Project Director of the NSF Undergraduate Research Program, and Mr. Ivars Gailans, who served as my assistant as an NSF Undergraduate Research Fellow. 